\title{
Differences in life-history traits in two clonal strains of the self-fertilizing fish, Rivulus marmoratus
}

Ma. Vivian Camacho Grageda ${ }^{1,2}$, Yoshitaka Sakakura ${ }^{3}$, Masako Minamimoto ${ }^{1}$ \& Atsushi Hagiwara ${ }^{1}$

${ }^{1}$ Graduate School of Science and Technology, Nagasaki University, 1-14 Bunkyo-machi, Nagasaki 852-8521, Japan

${ }^{2}$ Animal Biology Division, Institute of Biological Sciences, University of the Philippines at Los Baños, Laguna, Philippines

${ }^{3}$ Faculty of Fisheries, Nagasaki University, Nagasaki 852-8521, Japan (e-mail: sakakura@net.nagasaki-u.ac.jp)

Key words: mangrove killifish, clone, morphometry, fecundity.

\section{Synopsis}

We compared life-history traits such as fecundity, sex ratio, reproductive cycle, age at sexual maturity, embryonic period, egg size, early growth and morphology in two clonal strains (PAN-RS and DAN) of the mangrove killifish (Rivulus marmoratus) under constant rearing conditions. We found a positive relationship between growth and reproductive effort. Fecundity was significantly higher in the PAN-RS strain than in the DAN strain. Sex ratio was significantly different, with DAN producing more primary males than PAN-RS. Spawning and ovulation cycle did not clearly differ between the strains. PAN-RS showed a significantly higher growth rate than DAN from 0 to 100 days after hatching, however, age at sexual maturity, embryonic period, egg size, and morphometric and meristic characteristics (vertebral and fin-ray counts) did not differ between the two strains. The high fecundity of PAN-RS may provide an increased chance of offspring survival, while the attainment of sexual maturity at a smaller size in DAN may allow them to invest earlier in reproduction to increase breeding success. Variations in the life-history traits of PAN-RS and DAN may be adaptive strategies for life in their natural habitat, which consists of mangrove estuaries with a highly variable environment. 


\section{Introduction}

The individual traits of organisms and their interaction with the environment define the dynamics of populations, the structure of ecological communities, and the functioning of ecosystems (Tessier et al. 2000). Identifying trends in life-history traits and interpreting the adaptiveness of such variation has always been a challenge for biologists (Stearns 1992). It is generally believed that observed differences in life-history traits result from genotypic variation and/or from the effect on a plastic phenotype of variation in the environment (Belk 1995). One of the most important issues in evolutionary biology is defining whether differences in life-history traits can be attributed to genetic or environmental variation.

In the present study, we used two clonal strains of mangrove killifish (Rivulus marmoratus, Family Rivulidae) to identify differences in life-history traits and determine whether these variations are the result of genetic or environmental differences. The mangrove killifish is distributed from Southern Florida, U.S.A. to Brazil (Davis et al. 1990). It inhabits mangrove areas, which are exposed to alternate flooding and desiccation; thus, it is necessarily tolerant of a wide range of environmental conditions (Taylor 2000). There are three recognized sexual types: hermaphrodites, which have a marbled brownish color pattern usually including a caudal ocellus, and are capable of internal synchronous self-fertilization, and thus are capable of producing clonal lineages (Kallman \& Harrington 1964, Harrington \& Kallman 1968); primary males, which have an orange body coloration usually without caudal ocellus and remain male throughout their lives; and secondary males, which arise from hermaphrodites following the loss of female function (Harrington 1975). Based on these biological features, this species has been found suitable for studies on life-history traits, since hermaphrodites are able to produce clones. Subjecting mangrove killifish to controlled environmental conditions may allow the identification of possible reasons for observed variations in life-history traits, and may make this species an ideal organism for genotypic and phenotypic studies. The phenotypic plasticity of growth in mangrove killifish due to different environmental conditions has been previously documented (Lin \& Dunson 1995, 1999), and variation in certain characteristics such as age at sexual maturity, morphological anomalies, and low temperature induction of males between clones have been observed (Harrington 1971, Fisher 1997, as cited by Turner $2003^{1}$. However, to date there have been no reports on differences in life-history traits, such as fecundity, sex ratio, and growth in two clonal

\footnotetext{
1 Turner, B.J. 2003. The biology of Rivulus marmoratus, the only known self-fertilizing vertebrate species. The RivMar Webpage web site address:

http://www.biol.vt.edu/faculty/turner/rivmar/.
} 
strains reared under the same environmental conditions. In the present study, two strains of mangrove killifish, PAN-RS and DAN, were compared in terms of fecundity, sex ratio, reproductive cycle, age at sexual maturity, embryonic period, egg size, early growth, and morphometric and meristic features from 0 to 100 days after hatching (DAH), and we examined the relationships among these traits.

\section{Materials and methods}

Experimental fish and general rearing conditions

We used the two clonal strains, PAN-RS and DAN, obtained from Dr. W. P. Davis of the U.S. Environmental Protection Agency, Gulf Breeze, Florida, U.S.A. All PAN-RS individuals were the descendants of a single hermaphrodite collected near Bocas del Toro, Republic of Panama, in 1994 (previously thought to have originated in Florida, U.S.A,. by Sakakura \& Noakes 2000, Grageda et al. 2004), while all DAN individuals were the descendants of a single fish collected in Dangriga, Belize. These strains have been successfully reared for over 5 generations in our laboratory.

We reared larvae and juveniles individually in plastic cups $(5.8 \mathrm{~cm}$ in diameter) filled with $60 \mathrm{ml}$ of $17 \mathrm{ppt}$ artificial brackishwater maintained at $25 \pm 1^{\circ} \mathrm{C}$ and a photoperiod of 14L:10D. Fish were fed with fresh, newly hatched Artemia franciscana every two days (480 nauplii cup $^{-1}$ ).

We reared adult fish (> 5 months old) individually in specially designed rearing chambers (Zebrafish rearing system, AQUA Inc., Tokyo, Japan), which allowed the separation of eggs from the parent fish to avoid cannibalism. These chambers consisted of two 1-l plastic aquaria stacked on top of each other. The top aquarium was equipped with a net-like stainless steel bottom (2.2 mm mesh size), which was suspended $3.5 \mathrm{~cm}$ off the bottom of the lower aquarium, allowing eggs (about $1.7 \mathrm{~mm}$ in diameter) to pass through the mesh and settle in the lower aquarium, thus preventing the fish from eating their own eggs. The design of the rearing chamber was a modification of the reproduction chamber used for $R$. marmoratus by Koenig \& Chasar (1984). Fish were reared in a recirculating system with 17 ppt artificial brackishwater under controlled conditions ( $25 \pm 1^{\circ} \mathrm{C}, 14 \mathrm{~L}$ : 10D), and were fed 3 to 6 times week $^{-1}$ with pellets (Tetra Min, Tetra Werke Co., Mells, Germany) until satiation. Fish were given the same amount of food during each feeding.

We washed rearing cups and aquaria every two weeks, and the culture water in the cups was completely replaced after every washing. We checked eggs spawned in cups 
and in aquaria and collected prior to washing weekly and biweekly, respectively, using a glass pipette. We recorded the number of eggs spawned fish ${ }^{-1}$ and the dates of spawning individually from the year 2001.

Fecundity, age at sexual maturity, embryonic period and egg size

We calculated the fecundity (eggs week ${ }^{-1}$ ), age at sexual maturity, and embryonic period (number of days from spawning to hatching) of the PAN-RS and DAN strains based on the individual spawning record of the same fish used in the reproductive cycle experiment. We defined age at sexual maturity as the age when a live egg was first spawned (Stearns 1992). A total of 70 eggs (62 from PAN-RS and 8 from DAN) were collected from 32 adult fish (26 PAN-RS and 6 DAN), and the size of each egg was measured to the nearest $0.01 \mathrm{~mm}$ using a digital microscope (VH 6300, Keyence Corp., Osaka, Japan).

\section{Sex ratio}

For the comparison of sex ratio, we examined histologically the gonads of 196 fish (166 PAN-RS and $30 \mathrm{DAN}$ ) with ages ranging from 0 to $86 \mathrm{DAH}$. This number includes the 136 fish of the PAN-RS strain analyzed by Sakakura \& Noakes (2000), who used the same rearing conditions as in the present study. Whole fish were fixed in Bouin's fixative, embedded in paraffin, serially sectioned at $7 \mu \mathrm{m}$ transversely using a microtome, and finally stained with hematoxylin and eosin. We analyzed transverse sections of gonads for the absence or presence of sperm cells and egg cells using a microscope. When both oocytes and spermatocytes were present, the fish were classified as hermaphrodites, while when only spermatocytes were observed, the fish were classified as primary males.

\section{Reproductive cycle}

In order to compare the reproductive cycles of the two strains, we observed the spawning of 11 PAN-RS and 4 DAN adult fish (7-8 months old) at intervals of $3 \mathrm{~h}$ for 7 days. We recorded the number of eggs spawned at each observation time and the developmental stage of each embryo following the stages described by Koenig \& Chasar (1984). The time of ovulation of each egg was back-calculated according to the method of Koenig \& Chasar (1984). 


\section{Growth and morphometry}

We selected approximately 130 eggs, 65 of each strain at stage 32 (Koenig \& Chasar 1984), for this experiment. The embryo at stage 32 is in the final stage before hatching, characterized by opening and closing of the mouth with opercular movement, motile pectoral fins, ray formation in the dorsal and anal fins, and the appearance of pigmentation along the rays of the caudal and pectoral fins (Koenig \& Chasar 1984). The specimens were manually dechorionated by removal of the chorion/egg case using fine forceps to allow for the release of larvae, following the protocol described by Koenig \& Chasar (1984). As stated above, we sampled a total of 130 fish, 65 of each strain: 5 fish of each strain on 0 and 5 DAH; 10 fish on 10, 20, 30, and $60 \mathrm{DAH}$; and 15 fish on 100 DAH. The fish were anaesthetized with over 400 ppm of MS 222 (3-aminobenzoic acid ethyl ester, Sigma Chemical Co., St. Louis, MO, USA) and were fixed in $5 \%$ formalin solution. We examined the following morphological characteristics of the fish and measured them to the nearest $0.01 \mathrm{~mm}$ using a digital microscope: total length (TL), standard length (SL), preanal length (PAL), head length (HL), eye diameter (ED), snout length (SnL), gape size (GS $=\sqrt{ } 2 \mathrm{x}$ upper jaw length (UJL), Shirota 1970), body depth (BD), dorsal-fin length (DL), pectoral-fin length (PcL), anal-fin length (AL), and caudal-peduncle depth (CPD). Because of the large size of 60 and 100 DAH specimens, their TL and SL were measured to the nearest $0.1 \mathrm{~mm}$ using a caliper (Mitutoyo, Kawasaki, Japan). We measured all of the above characteristics following standard ichthyological methods (McAllister \& Smith 1978, Strauss \& Bond 1990). DL and AL were measured from the origin of the fin base to the posterior most portion of the fin margin.

\section{Meristic characteristics}

We examined the vertebral and fin-ray counts in 5 fish of each strain on $0,5,10,20,30$, 60, and $100 \mathrm{DAH}$. A clearing and staining technique was used following a modified version of the procedure described by Pothoff (1984). The number of vertebrae and fin rays was counted under a dissecting microscope.

\section{Statistical analysis}

The fecundity, age at sexual maturity, embryonic period, and egg size of [the] PAN-RS 
and DAN [strains] were compared by Student's $t$-test. We examined sex ratio (primary male:hermaphrodite) using a Chi-square test. To determine whether there was a diel difference in spawning and ovulation between strains, \% egg frequency data were initially subjected to arcsine transformation prior to paired $t$-test. The growth of each strain expressed as SL plotted over time (0-100 DAH) was fitted to the Gompertz growth curve equation: $Y=a \times e^{-\frac{-\left(x-x_{0}\right)}{b}}$ where $\mathrm{Y}=\mathrm{SL}, \mathrm{X}=\mathrm{DAH}, \mathrm{X}_{0}=$ initial instantaneous growth rate, $\mathrm{a}=$ slope, and $\mathrm{b}=\mathrm{y}$-intercept (Gamito 1998) using Sigma Plot version 8.0 (SPSS Inc., Chicago, IL, USA). Growth curve plots were linearly transformed and their slopes were compared using analysis of covariance (ANCOVA, Sokal \& Rohlf 1995). For each age group, meristic characteristics between strains were compared using the Student's $t$-test. To compare the morphometric characteristics between strains, we standardized values as a proportion of SL and plotted on the Y axis against SL, resulting in linear regressions described by the equation $\mathrm{Y}=\mathrm{a}+\mathrm{bX}$. The resulting slopes were compared using ANCOVA (Sokal \& Rohlf 1995).

\section{Results}

Fecundity (eggs week ${ }^{-1}$ ) was found to be significantly different between strains $(\mathrm{t}=$ -7.21, $\mathrm{P}<0.01$ ), with PAN-RS producing more eggs than DAN. However, age at sexual maturity was the same for both strains $(\mathrm{t}=1.47, \mathrm{P}=0.22)$. Our records of the duration of egg incubation of the two strains showed no significant difference $(t=-0.13, P=0.90)$, and egg size also did not differ significantly $(\mathrm{t}=0.81, \mathrm{P}=0.440)$ (Table 1 ).

Primary males occurred at a rate of $6.7 \%$ in the DAN strain, while PAN-RS had a lower primary male proportion at $0.6 \%\left(\chi^{2}=6.20, \mathrm{P}=0.01\right)$ (Table 2$)$.

Ovulation showed no diel rhythm in either strain, with t-values of -1.52 and 2.70 for PAN-RS and DAN, respectively $(\mathrm{P}>0.05)$. However, DAN frequently spawned in the dark $(\mathrm{t}=-5.00, \mathrm{P}=0.02)$, while PAN-RS did not show any clear diel rhythm in spawning $(\mathrm{t}=1.24, \mathrm{P}=0.243)$ (Figure 1$)$.

The growth of PAN-RS and DAN fitted closely into the Gompertz growth curve equation (Figure 2, $\mathrm{r}^{2}$ values were 0.97 and 0.96, respectively). PAN-RS showed a significantly faster growth rate than DAN (a-values were 16.9 and 16.4, respectively; ANCOVA; $n=65$ of each strain; degrees of freedom of adjusted means and error were 1 and 126, respectively; $\mathrm{F}=6.1923, \mathrm{P}<0.05$ ) (Figure 2). Growth in both strains indicated an inflection point at $20 \mathrm{DAH}$ (9.8 mm SL) (Figure 2). No significant differences were found between the strains in morphometric or meristic characteristics (Table 3, Figure 
3).

\section{Discussion}

In the present study, we demonstrated variations in the life-history traits of two clones of mangrove killifish reared under the same conditions. PAN-RS, the strain with higher growth, produced more eggs and had fewer primary males than DAN. Nevertheless, both strains showed the same age at sexual maturity, embryonic period, egg size, morphometry, and meristic characteristics. Additionally, the developmental stage at which our experiments were conducted was identical for both strains. Since both strains were exposed to the same environmental conditions, any observed difference in traits may be attributed to genetic differences.

The same inflection point in growth at $20 \mathrm{DAH}(9.8 \mathrm{~mm} \mathrm{SL})$ suggests the transformation of the mangrove killifish to the juvenile stage (Grageda et al. 2004).

\section{Positive relationship between growth and reproduction}

The PAN-RS strain had a higher number of reproducing entities (hermaphrodites) and higher fecundity than DAN. This suggests non-conformity to the life-history trade-off. The Principle of Allocation predicts that reproduction occurs at the expense of somatic growth as a direct consequence of energy being a limiting resource (Cody 1966, as cited by Glazier 1999). Therefore, a trade-off between growth and reproduction occurs and we can expect an inverse relationship between these two traits (Karasov 1986, Kozlowski 1992). However, in the present study, a positive relationship between growth and reproductive effort was observed. This can be explained using the four alternative reasons proposed by Glazier (1999). First, a negative correlation between pairs of life-history traits should not always be expected since masking effects, such as maternal effects and genotype-environment interaction may play a significant role. Second, since organisms are multi-trait systems, one should not expect them to respond similarly under any given conditions. Third, in the present study, the experimental fish were kept in highly favorable environmental conditions (followed the rearing protocol used by Koenig \& Chasar 1984 and Sakakura \& Noakes 2000), which allowed the fish to increase their growth without having any negative consequence on reproduction. And finally, individual differences in this species in terms of their ability to obtain resources may have resulted in higher growth in PAN-RS, that is, PAN-RS may have a more efficient means of converting energy for both growth and reproduction without showing 
any negative effects on other traits.

\section{Clonal heterogeneity as an adaptive strategy}

Although both strains had the same age at sexual maturity, the DAN strain attained sexual maturity at a smaller size than PAN-RS. The occurrence of smaller-sized individuals in DAN may be a strategy for them to be able to invest earlier in reproduction in order to maximize reproductive effort, and thus increase breeding success. Their small size may also be an advantage in their particular environment, that is, in an area like the estuary where organisms may experience high predation pressure due to constant exposure and visibility to possible predators such as crabs and other fish. Being small may thus allow them to escape easily from predators and hide in burrows. On the other hand, PAN-RS with their large size produce more eggs, and thus increase their chance of survival in their natural habitat, which is a highly unstable environment such as mangrove estuaries where chances of survival are expected to be rather low. The mangrove killifish inhabits mangrove estuaries and salt marsh intertidal zones, which have highly variable environmental conditions as they are potentially exposed to stressful conditions of hydroperiods, salinity, temperature, oxygen, and hydrogen sulphide (Lin \& Dunson 1999). Thus, genetic differences in the life-history traits of PAN-RS and DAN may be adaptive strategies and tactics suited to their life in a highly variable environment. As Lin \& Dunson (1999) point out, high levels of genetic variation among clones have to be maintained in order for them to adapt quickly to large variations in environmental conditions. The present results clearly demonstrate the potential of mangrove killifish to respond to a wide range of environmental conditions. This may also illustrate the temporal turnover of clonal composition, as has been previously suggested (Turner et al. 1990), with the small-sized strain possibly predominant during low tide levels when the risk of predators is high, and the large-sized strain dominating during high tide level.

Evidence of clonal heterogeneity in $R$. marmoratus has previously been documented. Harrington (1971) reports on differences in age at sexual maturity, the extent of senescent gender inversion, and the efficiency of low-temperature male induction among Harrington-Kallman clones, and states that, in response to short day seasons, Clone NA transformed from hermaphrodites into secondary males later than Clone DS. Differences in low-temperature male induction as well as morphological anomalies among seven other clones from Florida to Brazil have also been observed (Turner 2003 ${ }^{1}$ ). In the field, Turner et al. (1990, 1992a) used DNA fingerprinting to 
confirm both high levels of intrapopulation genetic heterogeneity and clonal variation in three Harrington-Kallman clones of Rivulus marmoratus. All of these studies demonstrate the high phenotypic and genetic variability of this species. Similar evidence of significant differences in characteristics between strains has also been reported for other fish species. Taniguchi et al. (1996) observed significant differences in body size and morphometric and meristic features in 4 clonal lines of Plecoglossus altivelis after rearing them for 9 months under common environmental conditions. Comparing two strains of Salvelinus alpinus held under comparable rearing conditions, Damsgard et al. (1999) report that both sex and strain influenced age at first maturity; additionally, the Hammerfest strain of charr was found to mature at a younger age and smaller body size than the Svalbard strain, suggesting genotypic differences between strains.

Genotypic differences in life-history traits

Variations in the life-history traits of mangrove killifish in response to different environmental conditions have been previously documented. Significant differences in the overall growth of hatchlings of this species have been reported to be due to differences in temperature, salinity, food rations and interactions among individuals (Lin \& Dunson 1999). Growth has been reported to be slow at low temperatures $\left(19^{\circ} \mathrm{C}\right)$, while at $26^{\circ} \mathrm{C}$, growth was higher in fish kept at 12 and 40 ppt salinity than in those exposed to 1 ppt (Lin \& Dunson 1999). Moreover, individuals whose parents were given high food rations showed faster growth during the first month than individuals whose parents were given low food rations (Lin \& Dunson 1999). Salinity and food have been found to have interactive effects on time to maturity, body size at maturity, and number of eggs produced, while low temperature delays sexual maturity (Lin \& Dunson 1995). At higher salinity, maturation occurs faster and is attained at a smaller size, however, this results in low hatching success of eggs and delayed hatching (Lin \& Dunson 1995). High food rations result in the production of more eggs (Lin \& Dunson 1995). All of these facts suggest clonal differences in ecologically relevant traits. However, in the present study, conditions such as temperature, salinity, illumination and food were maintained constant, and yet differences in fecundity, sex ratio and growth were detected between the PAN-RS and DAN strains. Since all specimens were exposed to similar rearing conditions (assuming that the genotype-environment interaction is negligible), this may indicate genotypic differences. Observed differences in growth between PAN-RS and DAN may be related to food intake and food intake regulation similar to what has been observed by Silverstein et al. (1999) between different genetic strains of channel catfish. 
In addition, the higher growth in a larger number of reproducing entities (hermaphrodites) may be similar to that found in a closely related species, Aphanius fasciatus, whose females are larger in size than the males; this has been interpreted to be a strategy characteristic of species in an unstable and variable environment (Leonardos \& Sinis 1999).

Low percentage of males: a transitory period leading to removal of males

Our results suggest that it is more costly to invest in males than in hermaphrodites, given that higher growth was observed in the strain with fewer males.

The low proportion of primary males observed in both strains (0.6 and 6.7\% for PAN-RS and DAN, respectively) may suggest a shift in population structure as part of the evolutionary process of complete removal of males from the population. This is in agreement with Charlesworth's view (as cited by Weeks et al. 2001) on the occurrence of androdioecy (populations comprised of males and hermaphrodites) being a transitory stage in the evolution of hermaphroditism from dioecy. The occurrence of hermaphrodites in the majority of the population and production of clonal lineages by self-fertilization makes it necessary to question the significance of male existence in such populations. Low proportions of males in $R$. marmoratus have in fact been previously reported, with ranges of less than $1 \%$ to $24 \%$ (Turner et al. 1992b, Davis et al. 1990, Cole \& Noakes 1997), and may indicate that males are becoming less important in the population. However, two major benefits of male occurrence in an otherwise all-hermaphroditic population have been suggested in a study on the Eulimnadia texana (freshwater shrimp) population: first, males are of benefit to the population due to increased allocation of reproductive resources to male function and reduction of inbreeding depression in offspring (Weeks et al. 2001), although in the case of mangrove killifish, this has been very difficult to study since the role of males is almost unknown; and second, clones may have evolved into a fixed point of male development and cannot evolve further toward a state of no males unless point chromosomal mutations or the induction of heterozygosity occurs in the clones. Moreover, the occurrence of higher numbers of males in the DAN strain may reflect the population origin of this strain; all members of the DAN strain are descendants of a single fish collected in Dangriga, Belize, the same place where an abundance of males (13.5-24 \%) has been reported (Turner et al., 1992b). Since the two strains come from different geographic locations (DAN from Belize and PAN-RS from Panama), the observed difference in sex ratio may be a unique feature of the groups in question. Since all the fish in the laboratory were 
maintained at $25 \pm 1^{\circ} \mathrm{C}$, the observed difference in the number of primary males may not be due to the artificial environment of the laboratory, as male induction has previously been reported to occur when embryos are incubated between 18 and $20^{\circ} \mathrm{C}$, (Harrington 1967, Harrington \& Kallman 1968). Additional field and laboratory studies are necessary to determine the influence of environmental conditions on male occurrence and to clarify its role in a population dominated by hermaphrodites. Moreover, the origin of $R$. marmoratus is not clearly understood, thus, its unique means of self-fertilization and reproduction may be a result of hybridization between two other species of Rivulus which have evolved rapidly, like the parthenogenetic teiid lizards of the North American Great Basin and the lacertid lizards of the Amazon, and the gynogenic Poecilia formosa from P. latipinna and P. mexicana crosses (Billy \& Crews 1986, Ryan et al. 1996, MacCulloch et al. 1997, Murphy et al. 2000).

Furthermore, higher fecundity in the higher growth strain may be a strategy to ensure propagation, given that they have a low number of males resulting in a low probability of mating. Although hermaphrodites of mangrove killifish can reproduce in the absence of males, this adaptation may have developed prior to the occurrence of hermaphroditism. The currently observed gender composition may be a transitory period leading to the eventual loss of males.

In the present study, we showed that PAN-RS, the strain with higher growth, produces more eggs and has fewer primary males than DAN. Our results also suggest that DAN attains sexual maturity at a smaller size than PAN-RS. Such variations in life-history traits observed in the two clonal strains of mangrove killifish may be related to survival and reproductive success (fitness) in their natural habitat. Since both strains were reared under the same environmental conditions, any differences in their traits may be genetic. However, to further understand the patterns of life-history variations, diversity among lineages in physiology and development should be studied (Stearns 1992). Since physiological functions mediate the relationship of organisms to their environment and most variation in life-history traits reflects phenotypic responses of individuals to environmental stresses, behavior and physiology should be integrated in any study on the problem of life-history variation (Ricklefs \& Wikelski 2002). Further investigation much be conducted to determine whether physiological and behavioral mechanisms explain variation in the life-history traits of mangrove killifish.

\section{Acknowledgements}

The authors would like to thank Drs. Kiyoshi Soyano and Atsuko Yamaguchi for allowing us the use of their laboratories for the histological analysis. The Ministry of 
Education, Culture, Sports, Science and Technology (Monbukagakusho) of Japan is gratefully acknowledged for the scholarship awarded to M.V.C.G. This study was supported financially by Japanese Grants-in-Aid for the Promotion of Scientific Research (13760145 and 15780135) to Y.S. and by the Nagasaki Prefecture Collaboration of Regional Entities for the Advancement of Technological Excellence, JST to Y.S. and A.H.

\section{References}

Belk, M.C. 1995. Variation in growth and age at maturity in bluegill sunfish: genetic or environmental effects? Journal of Fish Biology 47: 237-247.

Billy, A.J. \& D. Crews. 1986. The effects of sex steroid treatments on sexual differentiation in a unisexual lizard, Cnemidophorus uniparens. Journal of Morphology 187: 129-142.

Cole, K.S. \& D.L.G. Noakes. 1997. Gonadal development and sexual allocation in mangrove killifish, Rivulus marmoratus (Pisces: Atherinomorpha). Copeia 3: 596-600.

Damsgard, B., A.M. Arneser \& M. Jobling. 1999. Seasonal patterns of feed intake and growth of Hammerfest and Svalbard Arctic charr maturing at different stages. Aquaculture 171: 149-160.

Davis, W.P.D., S. Taylor \& B.J. Turner. 1990. Field observations of the ecology and habits of mangrove rivulus (Rivulus marmoratus) in Belize and Florida. Ichthyological Exploration of Freshwaters 1: 123-134.

Gamito, S. 1998. Growth models and their use in ecological modeling: an application to a fish population. Ecological Modelling 113: 83 - 94.

Glazier, D.S. 1999. Trade-offs between reproductive and somatic (storage) investments in animals: a comparative test of the Van Noordwijk and De Jong model. Evolutionary Ecology 13: 539-555.

Grageda, M.V.C., Y. Sakakura \& A. Hagiwara. (2004). Early development of the self-fertilizing mangrove killifish, Rivulus marmoratus, reared in the laboratory. Ichthyological Research 51: 309-315.

Harrington, R.W. Jr. 1967. Environmentally controlled induction of primary male gonochorists from eggs of the self-fertilizing hermaphroditic fish, Rivulus marmoratus Poey. Biological Bulletin 132: 174-199.

Harrington, R.W. Jr. 1971. How ecological and genetic factors interact to determine when self-fertilizing hermaphrodites of Rivulus marmoratus change into functional 
secondary males, with a reappraisal of the modes of intersexuality among fishes. Copeia 1971: 389-432.

Harrington, R.W. Jr. 1975. Sex determination and differentiation among uniparental homozygotes of the hermaphroditic fish Rivulus marmoratus (Cyprinodontidae: Atheriniformes). In R. Reinboth (ed.), Intersexuality in the Animal Kingdom, Springer, New York, pp. 249-262.

Harrington, R.W. Jr. \& K. Kallman. 1968. The homozygosity of clones of the self-fertilizing hermaphroditic fish Rivulus marmoratus Poey (Cyprinodontidae, Atheriniformes). American Naturalist 102: 337-343.

Kallman, K. \& R.W. Harrington, Jr. 1964. Evidence for the existence of homozygous clones in the self-fertilizing hermaphroditic teleost Rivulus marmoratus (Poey). Biological Bulletin 126: 101-114.

Karasov, W.H. 1986. Energetics, physiology, and vertebrate ecology. Trends in Ecology and Evolution 1: 101-104.

Koenig, C. \& M. Chasar. 1984. Usefulness of the hermaphroditic marine fish, Rivulus marmoratus, in carcinogenicity testing. National Cancer Institute Monographs 65: 15-33.

Kozlowski, J. 1992. Optimal allocation of resources to growth and reproduction: implications for age and size at maturity. Trends in Ecology and Evolution 7: 15-19.

Leonardos, I. \& A. Sinis. 1999. Population age and sex structure of Aphanius fasciatus Nardo, 1827 (Pisces: Cyprinodontidae) in the Mesolongi and Etolikon lagoons (W. Greece). Fisheries Research 40: 227-235.

Lin, H.C. \& W. Dunson. 1995. An explanation of the high strain diversity of a self-fertilizing hermaphroditic fish. Ecology 76: 593-605.

Lin, H.C. \& W. Dunson. 1999. Phenotypic plasticity in the growth of the self-fertilizing hermaphroditic fish Rivulus marmoratus. Journal of Fish Biology 54: 250-266.

MacCulloch, R.D., R.W. Murphy, L.A. Kupriyanova \& I.S. Darevsky. 1997. The Caucasian rock lizard Lacerta rostombekovi: a monoclonal parthenogenetic vertebrate. Biochemical Systematics and Ecology 25: 33-37.

McAllister, D. \& C. Smith. 1978. Mensurations morphologiques, dénombrements méristiques et taxonomie du coelacanthe, Latimeria chalumnae. Naturaliste Canadien 105: 63 - 76.

Murphy, R.W., J. Fu, R.D. MacCulloch, I.S. Darevsky \& L.A. Kupriyanova. 2000. A fine line between sex and unisexuality: the phylogenetic constraints on parthenogenesis in lacertid lizards. Zoological Journal of the Linnean Society 130: 527-549. 
Pothoff, T. 1984. Clearing and staining techniques. In H. Moser (ed.), Ontogeny and Systematics of Fishes, Allen Press Inc., Lawrence, pp. 35-37.

Ricklefs, R. \& M. Wikelski. 2002. The physiology/life history nexus. Trends in Ecology and Evolution 17: 462-467.

Ryan, M.J., L.A. Dries, P. Batra \& D. Hillis. 1996. Male mate preference in a gynogenetic species complex of Amazon mollies. Animal Behaviour 52: 1225-1226.

Sakakura, Y. \& D.L.G. Noakes. 2000. Age, growth, and sexual development in the self-fertilizing hermaphroditic fish Rivulus marmoratus. Environmental Biology of Fishes 59: 309-317.

Shirota, A. 1970. Studies on the mouth size of fish larvae. Bulletin of the Japanese Society of Scientific Fisheries 36: 353-368.

Silverstein, J.T., W.R. Wolters \& M. Holland. 1999. Evidence of differences in growth and food intake regulation in different genetic strains of channel catfish. Journal of Fish Biology 54: 607-615.

Sokal, R.R. \& F.J. Rohlf. 1995. Biometry: The Principles and Practice of Statistics in Biological Research, 2nd edition. W.H. Freeman and Company, New York. 887 pp.

Stearns, S.C. 1992. The Evolution of Life Histories. Oxford University Press, New York. 246 pp.

Strauss, R. \& C. Bond. 1990. Taxonomic methods: morphology. In M. Schreck \& P. Moyle (eds.), Methods for Fish Biology, American Fisheries Society, Maryland, pp. 109-140.

Taniguchi, N., M. Yamasaki, M. Takagi \& A. Tsujimura. 1996. Genetic and environmental variances of body size and morphological traits in communally reared clonal lines from gynogenetic diploid ayu, Plecoglossus altivelis. Aquaculture 140: 333-341.

Taylor, D.S. 2000. Biology and ecology of Rivulus marmoratus: new insights and a review. Florida Scientist 63: 242-255.

Tessier, A.J., M.A. Leibold \& J. Tsao. 2000. A fundamental trade-off in resource exploitation by Daphnia and consequences to plankton communities. Ecology 81: 826-841.

Turner, B.J., J.F. Elder, T.F. Laughlin \& W.P. Davis. 1990. Genetic variation in clonal vertebrates detected by simple-sequence DNA fingerprinting. Proceedings of the National Academy of Sciences, USA 87: 5653-5657.

Turner, B.J., J.F. Elder, T.F. Laughlin, W.P. Davis \& D.S. Taylor. 1992a. Extreme clonal diversity and divergence in populations of a selfing hermaphroditic fish. 
Proceedings of the National Academy of Sciences, USA 89: 10643-10647.

Turner, B.J., W.P. Davis \& D.S. Taylor. 1992b. Abundant males in populations of a selfing hermaphrodite fish, Rivulus marmoratus, from some Belize Cays. Journal of Fish Biology 40: 307-310.

Weeks, S.C., J.A. Hutchison \& N. Zucker. 2001. Maintenance of androdioecy in the freshwater shrimp, Eulimnadia texana: do hermaphrodites need males for complete fertilization? Evolutionary Ecology 15: 205-221. 
Figure 1

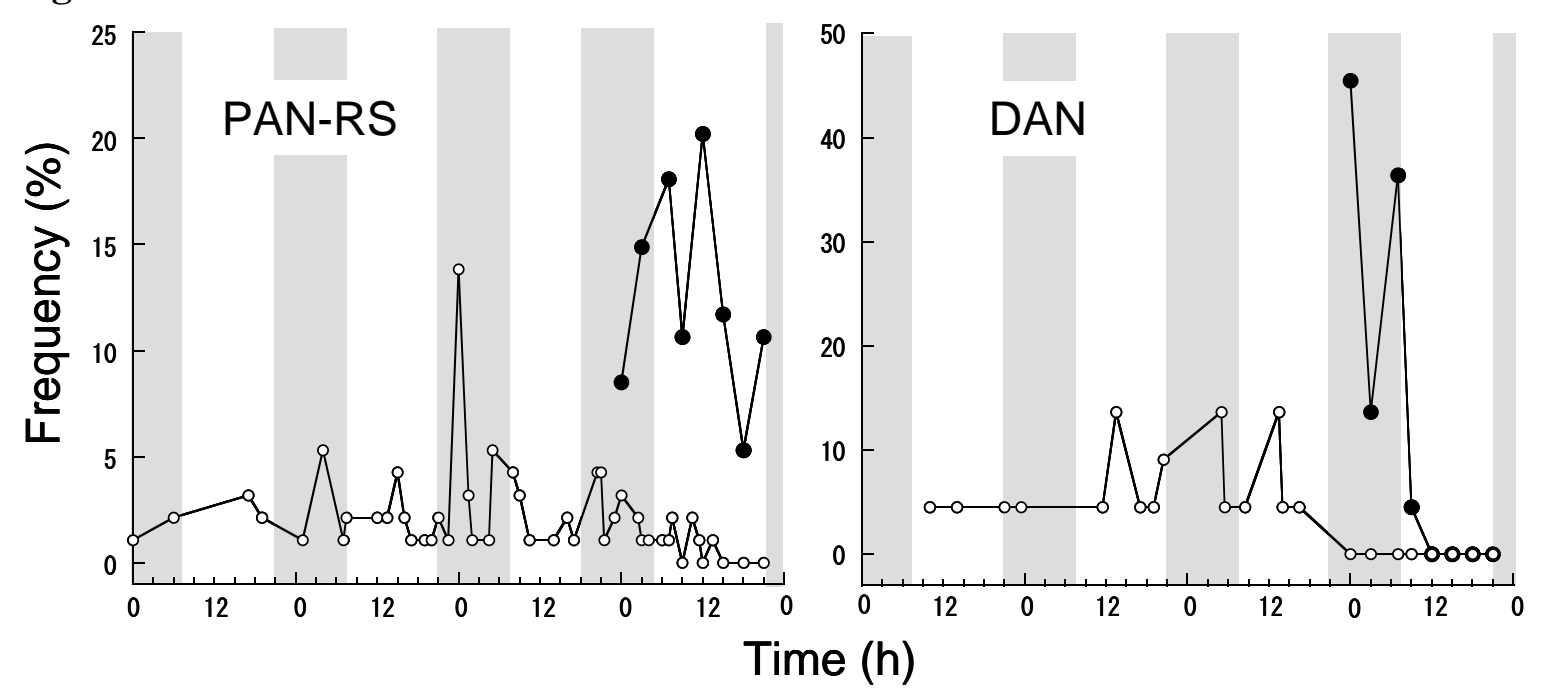

Figure 1. Ovulation (closed circles) and spawning cycles (open circles) of the PAN-RS and DAN strains ( $n=94$ eggs/11 fish and $n=22$ eggs/4 fish, respectively) monitored for 7 days at intervals of $3 \mathrm{~h}$. Shaded area in the graph represents the dark photoperiod.

\section{Figure 2}

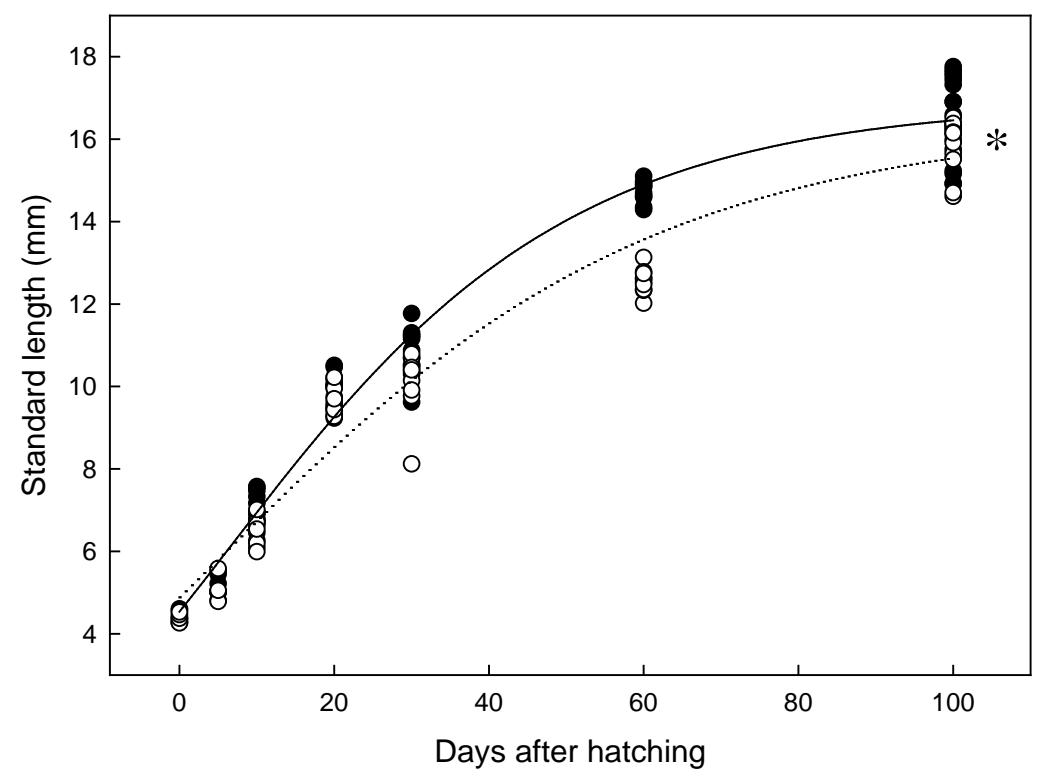

Figure 2. Comparison of growth curves between PAN-RS (closed circles) $\left(Y=16.9 e^{-\frac{-(x-6.9)}{25.6}}, \mathrm{r}^{2}=0.97, \mathrm{n}=65\right)$ and DAN (open circles) $\left(Y=16.4 e^{-\frac{-(X-6.3)}{32.5}}, \mathrm{r}^{2}=\right.$ 0.96; $n=65$ ). An asterisk denotes a statistically significant difference between the slopes of growth curves at $\mathrm{P}<0.05, \mathrm{~F}=6.1923$, ANCOVA. 


\section{Figure 3}
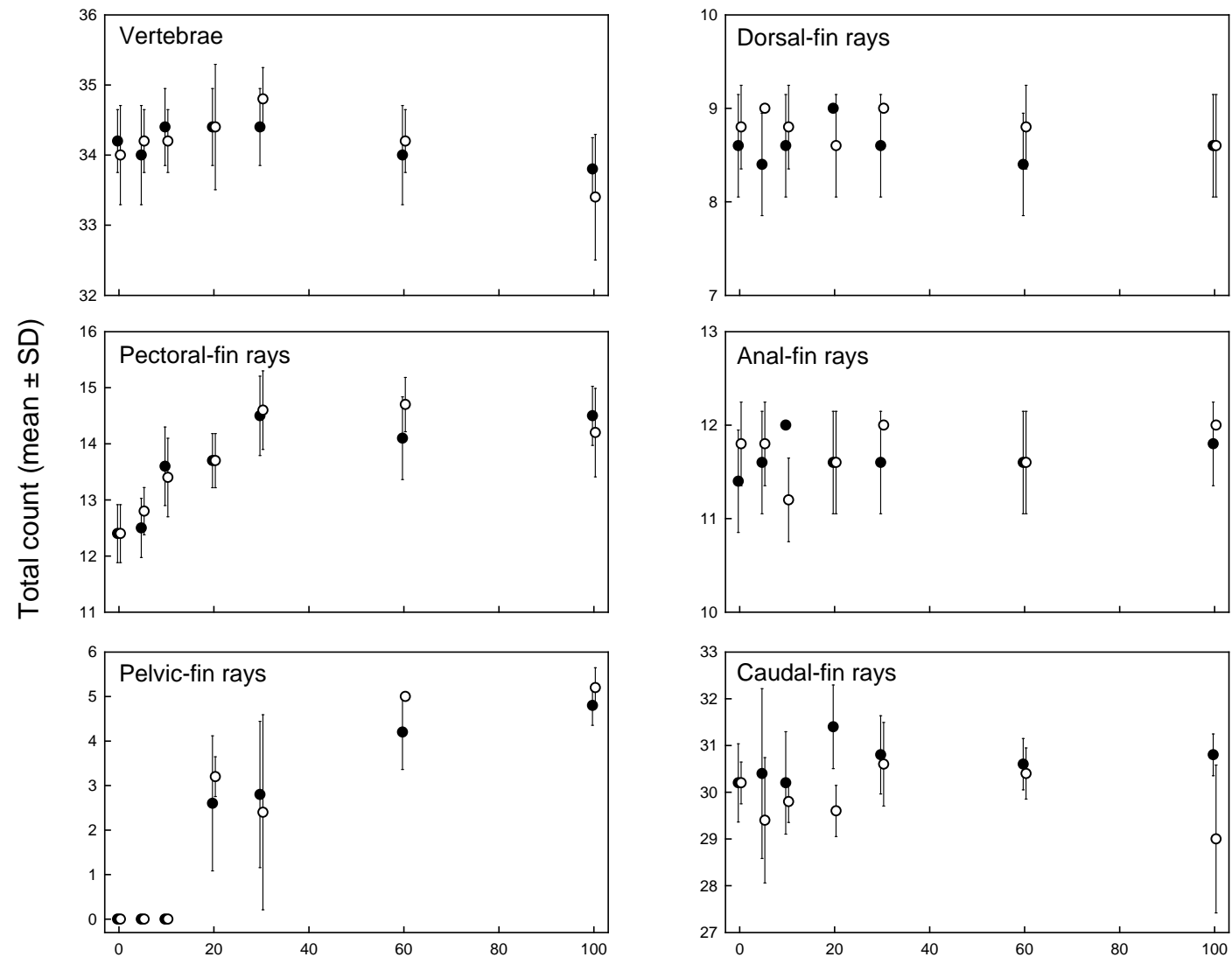

Days after hatching

Figure 3. Meristic characteristics (vertebral and fin-ray counts) of the PAN-RS and DAN strains of mangrove killifish. Each point indicates the total count (mean \pm SD) at each stage. 
Table 1. Fecundity, age at sexual maturity and egg size in PAN-RS and DAN strains.

\begin{tabular}{ccccc} 
Strain & $\mathrm{n}$ & $\begin{array}{c}\text { Fecundity } \\
(\text { eggs week }\end{array}$ (1) & $\begin{array}{c}\text { Age at sexual maturity } \\
(\mathrm{DAH} \pm \mathrm{SD})\end{array}$ & $\begin{array}{c}\text { Egg size }^{\mathrm{a}} \\
(\mathrm{mm} \pm \mathrm{SD})\end{array}$ \\
\hline PAN-RS & 11 & $2.2 \pm 0.6^{*}$ & $121.4 \pm 23.4$ & $1.7 \pm 0.1$ \\
DAN & 4 & $0.7 \pm 0.2$ & $146.7 \pm 31.5$ & $1.7 \pm 0.1$ \\
\hline
\end{tabular}

* Indicates significant difference at $\mathrm{t}=-7.21, \mathrm{P}<0.01$, Student's $t$-test.

${ }^{a}$ Represents data of 62 eggs from 26 fish and 8 eggs from 6 fish of the PAN-RS and DAN strains, respectively.

Table 2. Sex ratio (primary male:hermaphrodite) of the PAN-RS and DAN strains.

\begin{tabular}{cccc}
\hline Strain & $\mathrm{n}$ & Primary male & Hermaphrodite \\
\hline PAN-RS & $166^{*}$ & 1 & 165 \\
DAN & 30 & 2 & 28 \\
\hline
\end{tabular}

A Chi-square test yielded a significant difference at $\chi^{2}=6.20, P=0.013$.

* Includes 136 fish samples analyzed by Sakakura \& Noakes (2000).

Table 3. Morphometric parameters of the PAN-RS and DAN strains ( $n=65$ of each strain) showing $b$ (common slope of regression equation $\mathrm{Y}=\mathrm{a}+\mathrm{bX}$ ), $\mathrm{F}$, and $\mathrm{P}$ values resulting from ANCOVA.

\begin{tabular}{lccc}
\hline Morphometric variables & $\mathrm{b}$ & $\mathrm{F}$ & $\mathrm{P}$ \\
\hline Preanal length & 0.199675 & 1.5009 & $>0.1$ \\
Head length & -0.153074 & 0.3451 & $>0.1$ \\
Eye diameter & -0.195595 & 0.1664 & $>0.1$ \\
Snout length & 0.104589 & 0.0006 & $>0.1$ \\
Gape size & -0.00554 & 0.2606 & $>0.1$ \\
Body depth & -0.240052 & 0.8708 & $>0.1$ \\
Pectoral-fin length & 0.051313 & 0.0588 & $>0.1$ \\
Dorsal-fin length & 0.04955 & 0.7083 & $>0.1$ \\
Anal-fin length & 0.052297 & 0.0177 & $>0.1$ \\
& & & \\
Caudal-peduncle depth & 0.059774 & 0.0155 & $>0.1$ \\
\hline
\end{tabular}

